\title{
Exercise training modalities in patients with type 2 diabetes mellitus: a systematic review and network meta-analysis
}

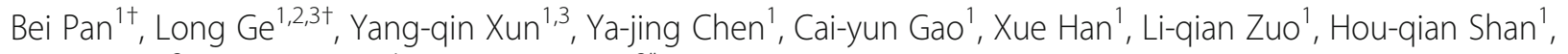
Ke-hu Yang ${ }^{3}$, Guo-wu Ding ${ }^{1}$ and Jin-hui Tian ${ }^{3^{*}}$

\begin{abstract}
Introduction: Current international guidelines recommend aerobic, resistance, and combined exercises for the management of type 2 diabetes mellitus (T2DM). In our study, we conducted a network meta-analysis to assess the comparative impact of different exercise training modalities on glycemic control, cardiovascular risk factors, and weight loss in patients with T2DM.
\end{abstract}

Methods: We searched five electronic databases to identify randomized controlled trials (RCTs) that compared the differences between different exercise training modalities for patients with T2DM. The risk of bias in the included RCTs was evaluated according to the Cochrane tool. Network meta-analysis was performed to calculate mean difference the ratio of the mean and absolute risk differences. Data were analyzed using R-3.4.0.

Results: A total of 37 studies with 2208 patients with T2DM were included in our study. Both supervised aerobic and supervised resistance exercises showed a significant reduction in HbA1c compared to no exercise $(0.30 \%$ lower, $0.30 \%$ lower, respectively), however, there was a less reduction when compared to combined exercise $(0.17 \%$ higher, $0.23 \%$ higher). Supervised aerobic also presented more significant improvement than no exercise in fasting plasma glucose (9.38 mg/dl lower), total cholesterol (20.24 mg/dl lower), triacylglycerol (19.34 mg/dl lower), and low-density lipoprotein cholesterol $(11.88 \mathrm{mg} / \mathrm{dl}$ lower). Supervised resistance showed more benefit than no exercise in improving systolic blood pressure $(3.90 \mathrm{mmHg}$ lower]) and total cholesterol $(22.08 \mathrm{mg} / \mathrm{dl}$ lower]. In addition, supervised aerobic exercise was more powerful in improving $\mathrm{HbA1c}$ and weight loss than unsupervised aerobic (HbA1c: 0.60\% lower; weight loss: $5.02 \mathrm{~kg}$ lower) and unsupervised resistance (HbA1c: 0.53\% lower) exercises.

Conclusion: Compared with either supervised aerobic or supervised resistance exercise alone, combined exercise showed more pronounced improvement in $\mathrm{HbA1c}$ levels; however, there was a less marked improvement in some cardiovascular risk factors. In terms of weight loss, there were no significant differences among the combined, supervised aerobic, and supervised resistance exercises.

Trial registration: Our study protocol was registered with the International Prospective Register of Systematic Reviews (PROSPERO); registration number: CRD42017067518.

Keywords: Type 2 diabetes mellitus, Exercise training, Glycemic control, Cardiovascular risk factors, Weight loss, Frequentist network meta-analysis

\footnotetext{
*Correspondence: tjh996@163.com

${ }^{\dagger}$ Bei Pan and Long Ge contributed equally to this work.

${ }^{3}$ Evidence-Based Medicine Center, School of Basic Medical Sciences, Lanzhou

University, No. 199, Dong gang West Road, Chengguan District, Lanzhou

City, Gansu province, China

Full list of author information is available at the end of the article
}

(c) The Author(s). 2018 Open Access This article is distributed under the terms of the Creative Commons Attribution 4.0 International License (http://creativecommons.org/licenses/by/4.0/), which permits unrestricted use, distribution, and reproduction in any medium, provided you give appropriate credit to the original author(s) and the source, provide a link to the Creative Commons license, and indicate if changes were made. The Creative Commons Public Domain Dedication waiver (http://creativecommons.org/publicdomain/zero/1.0/) applies to the data made available in this article, unless otherwise stated. 


\section{Background}

Diabetes affects approximately 382 million adults worldwide, and is predicted to increase to 439 million adults by $2030[1,2]$. Type 2 diabetes mellitus (T2DM) accounts for $85-95 \%$ of all diabetes cases in the world [2]. A sedentary lifestyle is considered as one of the major risk factors for T2DM and its complications [3]. Maintaining an appropriate level of physical activity is an effective strategy for T2DM management $[4,5]$.

Most patients with T2DM display dyslipidemia, hypertension and hyperinsulinemia, which are associated with metabolic syndrome and will lead to an increased risk of premature cardiovascular disease [6]. Comorbid conditions and complications are considered to determine the quality of life of patients with T2DM [7, 8]. Improved muscular and cardiorespiratory fitness are associated with reduced mortality rates [9-11]. Studies have shown that resistance training exercise can increase muscular strength and improve the control of blood glucose and HbA1c levels [12]. Aerobic exercise can also increase cardiorespiratory fitness and improve the control of blood glucose and HbA1c levels in patients with T2DM $[13,14]$.

Physical activity has been recommended as an important non-pharmacological therapeutic strategy for the management of T2DM by some major international organizations in this field [15]. Current national and international guidelines recommend aerobic and resistance exercise training for T2DM patients [16-19]. A combination of aerobic exercise and resistance exercise (combined exercise) has been recommended by the European Society of Cardiology [16], American College of Sports Medicine [17], Belgian Physical Therapy Association [18], and Exercise and Sports Science Australia [19]. Thus, multiple exercise training modalities have been recommended by different international organizations. The latest Canadian guideline [20] recommends supervised exercise as an effective modality for improving glycemic control, and weight loss. Different training modalities such as aerobic exercise, resistance exercise, combined exercise, and flexibility training are recommended. The recommendations are thus inconclusive and some bodies specifies supervised exercise in their recommendations.

Several randomized controlled trials (RCTs) and systematic reviews have been conducted to investigate the impact of aerobic or resistance exercise on glycemic control, cardiovascular risk factors and muscle strength in T2DM patients [21-24]. However, it is difficult to determine the superiority of different physical activities using RCTs or pairwise meta-analysis. Network meta-analysis has become increasingly popular to evaluate healthcare interventions, since it allows for estimation of the relative effectiveness among all interventions and rank ordering of the interventions even if head-to-head comparisons are lacking [25].
In the current study, we aimed to compare different exercise training modalities in the improvement of glycemic control, weight loss, and cardiovascular risk factors for patients with T2DM using Frequentist network meta-analysis.

\section{Methods and analysis Registration}

Our study protocol was registered with the International Prospective Register of Systematic Reviews (PROSPERO); registration number: CRD42017067518.

\section{Search strategy}

Searches of the PubMed, EMBASE, and Cochrane Central Register of Controlled Trials (CENTRAL) databases were conducted in April 2017. The references of included articles and relevant systematic reviews and meta-analyses were tracked for additional studies. There were no restrictions in terms of the year of publication or publication status. Search terms included: random*, type 2 diabetes, exercise, aerobic exercise, resistance exercise and combined exercise. The search strategy is shown in Additional file 1: Appendix 1.

\section{Inclusion criteria}

Type of participants: We included studies enrolling participants with T2DM aged $\geq 18$ years. Studies including patients with other chronic diseases, children, adolescents or pregnant women were excluded [26].

Type of design: Randomized controlled trials (RCTs).

Type of interventions: We focused on the following eight exercise training modalities: supervised aerobic exercise, unsupervised aerobic exercise, anaerobic exercise, supervised resistance exercise, unsupervised resistance exercise, combined exercise, flexibility exercise, and no exercise. The definition of each intervention is shown in Additional file 1: Appendix 2.

Type of outcomes: Outcomes of interest included glycemic control [including $\mathrm{HbA1c}$, fasting plasma glucose (FBG)], weight loss, and cardiovascular risk factors [total cholesterol (TC), low-density lipoprotein cholesterol (LDL), high-density lipoprotein cholesterol (HDL), triacylglycerol (TG), diastolic blood pressure (DBP) and systolic blood pressure (SBP).

\section{Study selection}

ENDNOTE X7 literature management software was used to manage the literature search records. To ensure high inter-rater reliability among the reviewers, a pilot-literature selection was performed.

Two reviewers independently screened the titles and abstracts of all the retrieved bibliographic records according to our eligibility criteria. Any studies with the potential to meet our inclusion criteria and conflicted studies were 
subjected to full-text evaluation. Any conflict was resolved by a third reviewer.

\section{Data extraction}

Paired reviewers independently extracted the following data of interest: the first author, year of publication, country, study design (RCT), sample, diagnostic criteria for T2DM, study period, mean age, median weight, body mass index (BMI), mean baseline HbA1c, FBG, weight, LDL, HDL, TC, TG, SBP, and DBP, details of interventions, diabetes duration. Data were presented as the mean \pm standard deviation (SD) at the end of the study; if values at the end of study were not available, they were imputed according to the Cochrane Handbook [27].

\section{Risk of bias of individual studies}

The risk of bias in the included RCTs was assessed according to the Cochrane Handbook version 5.1.0 [27], including the method of adequate sequence generation, allocation concealment, blinding of participants and personnel, incomplete outcome data, selective reporting, and other sources of bias (e.g. early trial termination, extreme baseline imbalance). We classified the methodological quality as having a low, high, or unclear risk of bias. The risk of bias assessment was completed independently by two reviewers, and conflict was resolved by a third reviewer.

\section{Data analysis}

We used the 'netmeta' version 0.9-2 of R-3.4.0 software to perform a Frequentist network meta-analysis [28]. The function of 'networkplot' function of STATA 15.1 (College Station, Texas 77,845 USA) was used to draw generate network plots to describe and present the geometry of different form of exercise. We used nodes to represent different interventions and edges to represent the head-to-head comparisons between interventions. The 'decomp.design' function was performed to assess the homogeneity in the whole network, the homogeneity within designs, and the homogeneity/consistency between designs. A node-splitting method was used to evaluate the inconsistency between direct and indirect comparisons [29]. Treatment ranking was calculated according to P-scores, which were based solely on the point estimates and standard errors of the network estimates. These scores measure the extent of certainty that a treatment is better than another treatment, averaged over all competing treatments [30].

A random effects network meta-analysis was performed to calculate pooled estimates and 95\% confidence intervals $(95 \% \mathrm{CI})$. In general, when the same measurement unit was used among studies for our outcomes of interest, the mean difference (MD) was considered as treatment effects to analyze the results or the standardized mean difference (SMD) was considered.
However, when studies in meta-analyses were weighted by the inverse of the variance of the effect measure, the pooled SMD was associated with unfavorable negative bias. In our analysis, we used the ratio of the mean (RoM) to measure the treatment effect in the intervention group relative to that in the control group [31]; this value accounted for the baseline difference being roughly comparable through different measurement units. Then, we calculated and presented absolute risk differences (ARD) by using RoM and the baseline risk of no exercise.

We also planned to perform subgroup analyses to observe the discrepancy for a specific population. The subgroup factors were as follows: exercise length (longer term vs. shorter term, with 6 months used as a cut-off point based on the previous reviews) [32], type of patients (sedentary patients vs. non-sedentary patients, based on the reporting of the original studies), age (younger vs. older population, using age 60 years as a cut-off point based on World Health Organization report [33], and the duration of diabetes (longer duration vs. shorter duration, using the median duration of diabetes reported in the included studies as a cut-off point).

\section{Results}

\section{Literature selection}

A total of 3966 studies were initially identified in this study. After reviewing the title and abstract, 75 studies were selected for further review. Of these, 41 were excluded (11 did not report the data of interest, 4 did not investigate T2DM, 3 were not RCTs, 12 did not meet our inclusion criteria, 6 did not report the outcomes included in our review, and 4 were non-English language). Finally, 37 studies met our inclusion criteria [34-70]. The detailed selection process is described in Fig. 1.

\section{Characteristics of the included studies}

The characteristics of the included studies are presented in Table 1. A total of 2208 patients with T2DM were included into our network meta-analysis. The majority of the included studies consists of two arms and was published between 1998 and 2014. The study period of 26 included studies was less than 6 months. The mean age of patients ranged from 55.1 to 71.9 years. A total of 1079 patients enrolled in 14 studies reported previous sedentary behavior.

We included the following eight exercise training modalities in our network meta-analysis (Fig. 2): supervised aerobic, unsupervised aerobic, anaerobic, supervised resistance, unsupervised resistance, combined exercise, flexibility training, and no exercise.

\section{Results of risk of bias}

The results of risk of bias are provided in Additional file 1: Appendix 3. Two articles were judged to have a 


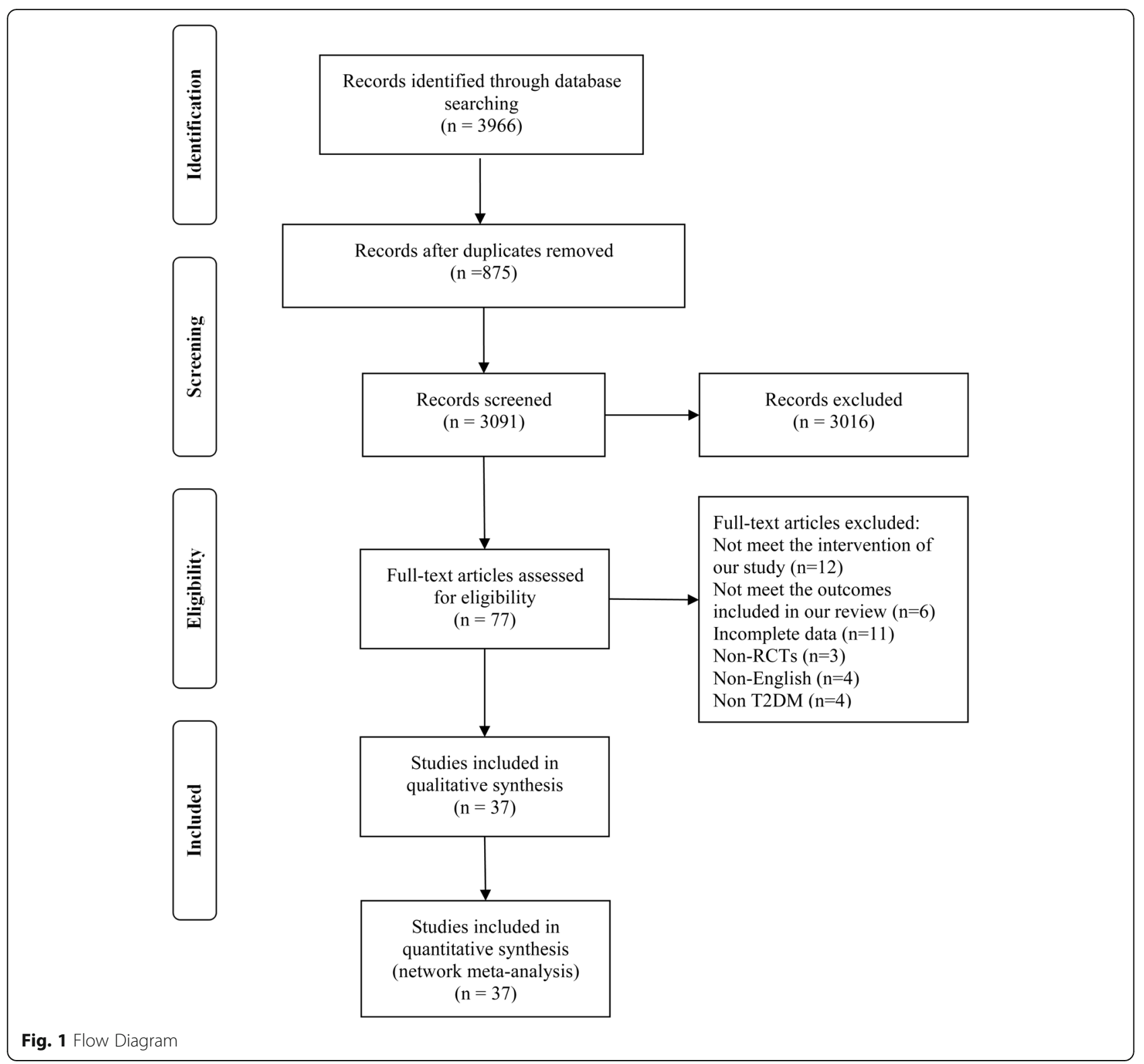

high risk of bias in adequate sequence generation [40, 66]. Fifteen articles showed unclear risk of bias [37-39, $41,42,47,50,55,57,5863-65,67,68]$ and six showed high risk of bias $[40,51,52,61,62,66]$ in adequate allocation concealment. Blinded outcome assessment was reported in 10 studies [39, 44-47, 49, 53, 59, 60, 69]. In terms of incomplete outcome data, four studies were judged as high risk $[36,54,66,69]$ and three were unclear risk [35, 43, 48, 67]. Eleven studies showed unclear risk of bias in selective reporting [34, 35, 40, 41, $45,47,50,53,59,60,66]$, and 16 showed unclear risks of other biases [38, 42, 44, 45, 47, 48, 50, 55, 57, 59, 60, $62,63,65,67,69]$.

\section{Network meta-analysis}

\section{Glycemic control}

Twenty-six studies involving 1729 patients reported HbA1c and FBG. Of these, two studies $[64,65]$ were not included in our network meta-analysis of HbA1c and our study [43] was not included in our network meta-analysis of FBG due to the imbalance in the baselines in these studies.

Compared to no exercise, combined exercise $(-0.53$, $95 \%$ CI: $-0.68 \%$ to $-0.45 \%)$, supervised aerobic $(-0.30$, $95 \%$ CI: $-0.60 \%$ to $-0.45 \%)$, supervised resistance $(-0.30$, $95 \%$ CI: $-0.38 \%$ to $-0.15 \%)$, and flexibility training $(-0.60$, $95 \%$ CI: $-1.05 \%$ to $-0.15 \%)$ showed significant reduction in 
Table 1 Characteristic of included studies

\begin{tabular}{|c|c|c|c|c|c|c|c|}
\hline Author & Country & $\begin{array}{l}\text { Type of } \\
\text { patients }\end{array}$ & $\begin{array}{l}\text { Study } \\
\text { period } \\
\text { (months) }\end{array}$ & Interventions & $\begin{array}{l}\text { Sample } \\
\text { N/F/M }\end{array}$ & $\begin{array}{l}\text { Mean age } \\
\text { (years) }\end{array}$ & Exercise prescription \\
\hline \multirow[t]{2}{*}{ Kwon 2010} & Korea & NA & 3 & unsupervised RE & $13 /-/-$ & $55.7 \pm 6.2$ & 40-50\% 1RM; each session 40 min \\
\hline & & & & no exercise & $15 /-/-$ & $57.0 \pm 8.0$ & NA \\
\hline \multirow[t]{2}{*}{ Okada 2010} & Japan & NA & 3 & combined & $21 / 11 / 10$ & $61.9 \pm 8.6$ & 3-5 times weekly; each session $60 \mathrm{~min}$ \\
\hline & & & & no exercise & $17 / 6 / 11$ & $64.5 \pm 5.9$ & NA \\
\hline \multirow[t]{2}{*}{ Aylin 2009} & Turkey & NA & 2 & combined & 18/3/15 & $51.39 \pm 2.02$ & $\begin{array}{l}\text { RT: } 50 \% \text { 1RM; each session } 30 \text { min; AT: } \\
\text { walking with moderate intensity }\end{array}$ \\
\hline & & & & no exercise & $18 / 6 / 12$ & $56.06 \pm 1.48$ & NA \\
\hline \multirow[t]{2}{*}{ Kenneth 2013} & Switzerland & Sedentary & 3 & supervised AE & $20 /-/-$ & $68.5 \pm 0.9$ & $\begin{array}{l}\text { moderate to vigorous intensity; } 3 \text { times } \\
\text { weekly; each session } 60 \text { min. }\end{array}$ \\
\hline & & & & anaerobic & $20 /-/-$ & $70 \pm 0.8$ & $\begin{array}{l}\text { minimal aerobic component; } \\
\text { nonstrenuous strength training }\end{array}$ \\
\hline \multirow[t]{4}{*}{ Larose 2011} & Canada & Sedentary & 6 & combined & $64 / 24 / 40$ & $53.5 \pm 7.3$ & NA \\
\hline & & & & supervised AE & $60 / 21 / 39$ & $53.9 \pm 6.6$ & $60 \%$ VO2peak; $75 \%$ heart rate; 3 times weekly \\
\hline & & & & supervised RE & $64 / 24 / 40$ & $54.7 \pm 7.5$ & 2-3 times weekly; each exercise $2-3$ sets \\
\hline & & & & no exercise & $63 / 22 / 41$ & $54.8 \pm 7.2$ & $\begin{array}{l}\text { full aerobic training programme plus } \\
\text { the full resistance training }\end{array}$ \\
\hline \multirow[t]{2}{*}{ Stolinskia 2008} & UK & NA & 6 & supervised AE & $8 / 4 / 4$ & $59 \pm 3$ & $\begin{array}{l}\text { 60-85\%VO2peak; } 4 \text { times weekly; } \\
\text { supervised by trainer }\end{array}$ \\
\hline & & & & unsupervised AE & $9 / 4 / 5$ & $55 \pm 3$ & $\begin{array}{l}60-85 \% \text { VO2peak; } 4 \text { times weekly Only } \\
\text { contact trainers initially }\end{array}$ \\
\hline \multirow[t]{2}{*}{ Arslan 2014} & Turkey & NA & 3 & supervised AE & $31 / 14 / 17$ & $53.5 \pm 6.5$ & 75\% maximum heart rate; each session 45 min \\
\hline & & & & no exercise & $33 / 19 / 14$ & $54.0 \pm 9.4$ & NA \\
\hline \multirow[t]{2}{*}{ Yavari 2010} & Iran & NA & 4 & unsupervised AE & $30 / 14 / 16$ & $49.76 \pm 6.56$ & $\begin{array}{l}60-85 \% \text { VO2peak; } 50 \% \text { maximum heart } \\
\text { rate;each session } 4 \text { min }\end{array}$ \\
\hline & & & & no exercise & $30 / 18 / 12$ & $49.86 \pm 6.39$ & NA \\
\hline \multirow[t]{2}{*}{ Shenoy 2010} & India & NA & 2 & unsupervised $\mathrm{AE}$ & 20/5/15 & $53.15 \pm 4.4$ & $\begin{array}{l}\text { moderate intensity; } 50-70 \% \text { maximum } \\
\text { heart rate; } 150 \text { min weekly }\end{array}$ \\
\hline & & & & no exercise & $20 / 6 / 14$ & $51 \pm 5.4$ & NA \\
\hline \multirow[t]{3}{*}{ KU 2010} & Korea & NA & 3 & supervised RE & $13 / 13 / 0$ & $55.7 \pm 6.2$ & 40-45\% maximum capacity; 5 times weekly; \\
\hline & & & & supervised $A E$ & $15 / 15 / 0$ & $55.7 \pm 6.2$ & Moderate intensity; 5 times weekly; \\
\hline & & & & no exercise & $16 / 16 / 0$ & $57.8 \pm 8.1$ & NA \\
\hline \multirow[t]{2}{*}{ Belli 2011} & Brazil & Sedentary & 3 & supervised $A E$ & $9 / 9 / 0$ & $55.9 \pm 2.2$ & $\begin{array}{l}\text { Time of walking increase from } 20 \text { min to } \\
50 \text { min }\end{array}$ \\
\hline & & & & no exercise & 10/10/0 & $53.4 \pm 2.3$ & NA \\
\hline \multirow[t]{2}{*}{ Dunstan 1998} & Australia & Sedentary & 2 & supervised RE & $11 / 3 / 8$ & $50.3 \pm 2.0$ & 50-55\% 1RM; each session 60 min; \\
\hline & & & & no exercise & $10 / 5 / 5$ & $51.1 \pm 2.2$ & NA \\
\hline \multirow[t]{4}{*}{ Reid 2010} & Canada & NA & 6 & combined & $57 / 19 / 38$ & $53.3 \pm 7.2$ & $\begin{array}{l}\text { full aerobic program plus the full resistance } \\
\text { program }\end{array}$ \\
\hline & & & & supervised RE & $58 / 20 / 38$ & $54.7 \pm 7.6$ & $\begin{array}{l}8 \text { different exercises on weight machines } \\
\text { each session; } 3 \text { times weekly }\end{array}$ \\
\hline & & & & supervised AE & $51 / 18 / 33$ & $53.8 \pm 6.4$ & 60-75\% maximum heart rate; 3 times weekly \\
\hline & & & & no exercise & $52 / 19 / 33$ & $55.2 \pm 6.9$ & NA \\
\hline \multirow[t]{3}{*}{ Sigal 2007} & Canada & NA & 6 & combined & $64 / 24 / 40$ & $53.5 \pm 7.3$ & $\begin{array}{l}\text { full aerobic program plus the full } \\
\text { resistance program }\end{array}$ \\
\hline & & & & supervised RE & $60 / 21 / 30$ & $53.9 \pm 6.6$ & $\begin{array}{l}7 \text { different exercises on weight } \\
\text { machines each session }\end{array}$ \\
\hline & & & & supervised AE & $64 / 24 / 40$ & $54.7 \pm 7.5$ & $\begin{array}{l}60-75 \% \text { maximum heart rate; } \\
3 \text { times weekly }\end{array}$ \\
\hline
\end{tabular}


Table 1 Characteristic of included studies (Continued)

\begin{tabular}{|c|c|c|c|c|c|c|c|}
\hline Author & Country & $\begin{array}{l}\text { Type of } \\
\text { patients }\end{array}$ & $\begin{array}{l}\text { Study } \\
\text { period } \\
\text { (months) }\end{array}$ & Interventions & $\begin{array}{l}\text { Sample } \\
\text { N/F/M }\end{array}$ & $\begin{array}{l}\text { Mean age } \\
\text { (years) }\end{array}$ & Exercise prescription \\
\hline & & & & no exercise & $63 / 22 / 41$ & $54.8 \pm 7.2$ & NA \\
\hline \multirow[t]{2}{*}{ Choi 2012} & Korea & Sedentary & 3 & no exercise & $37 /-/-$ & $55.0 \pm 6.0$ & NA \\
\hline & & & & unsupervised $A E$ & $38 /-/-$ & $53.8 \pm 7.2$ & $\begin{array}{l}\text { Moderate intensity; each session } \\
60 \text { min; } 5 \text { times weekly }\end{array}$ \\
\hline \multirow[t]{3}{*}{ Arora 2007} & India & NA & 2 & no exercise & $10 / 4 / 6$ & $58.4 \pm 1.8$ & NA \\
\hline & & & & supervised RE & $10 / 6 / 4$ & $49.6 \pm 5.2$ & $\begin{array}{l}60 \% 1 \mathrm{RM} ; 3 \text { sets of } 10 \text { repetitions of } \\
7 \text { exercise each session; }\end{array}$ \\
\hline & & & & unsupervised $A E$ & $10 / 4 / 6$ & $52.2 \pm 9.3$ & 2 times weekly; each session 30 min \\
\hline \multirow[t]{4}{*}{ Oliveira 2012} & Brazi & NA & 3 & $\begin{array}{l}\text { flexibility } \\
\text { training }\end{array}$ & $12 / 8 / 4$ & $53.42 \pm 9.82$ & perform stretching exercises \\
\hline & & & & supervised AE & $11 / 6 / 5$ & $52.09 \pm 8.71$ & $\begin{array}{l}\text { Cycling time increase from } 20 \mathrm{~min} \\
\text { to } 50 \mathrm{~min}\end{array}$ \\
\hline & & & & supervised RE & $10 / 6 / 4$ & $54.10 \pm 8.94$ & $\begin{array}{l}50 \% 1 \mathrm{RM} ; 4 \text { sets of } 8 \text { to } 12 \text { repetitions } \\
\text { of } 7 \text { exercise }\end{array}$ \\
\hline & & & & combined & $10 / 6 / 4$ & $57.90 \pm 9.82$ & $\begin{array}{l}\text { same intensity and half the volume of } \\
\text { that in the AT and ST groups }\end{array}$ \\
\hline \multirow[t]{4}{*}{ Jennings 2009} & Canada & Sedentary & 6 & combined & 19/6/13 & $54.48 \pm 7.68$ & 3 times weekly \\
\hline & & & & supervised RE & $18 / 7 / 11$ & $52.84 \pm 7.54$ & 3 times weekly \\
\hline & & & & supervised AE & $13 / 7 / 6$ & $55.35 \pm 7.49$ & 3 times weekly \\
\hline & & & & no exercise & $22 / 11 / 11$ & $56.33 \pm 6.91$ & NA \\
\hline \multirow[t]{2}{*}{ Cauza 2005} & Australia & NA & 4 & unsupervised RE & $22 / 11 / 11$ & $56.4 \pm 1.1$ & $\begin{array}{l}3 \text { sets per muscle group per week; } \\
3 \text { times weekly }\end{array}$ \\
\hline & & & & unsupervised AE & $17 / 8 / 9$ & $57.9 \pm 1.4$ & $60 \% \mathrm{VO}_{2 \text { max }} ; 3$ times weekly \\
\hline \multirow[t]{2}{*}{ Franciele 2013} & USA & NA & 7 days & unsupervised AE & $7 / 2 / 5$ & $56 \pm 2$ & 70\% peak heart rate; each session 40 min \\
\hline & & & & combined & $7 / 2 / 5$ & & $\begin{array}{l}20 \text { min AT at } 70 \% \text { peak heart rate } \\
\text { and } 4 \text { RT } 3 \text { sets of } 12 \text { repetitions } \\
\text { at } 65 \% 1 \mathrm{RM}\end{array}$ \\
\hline \multirow[t]{2}{*}{ Cheung 2009} & Australia & Sedentary & 4 & unsupervised RE & 20/13/7 & $59 \pm 8.7$ & 5 times weekly; each session 30 min \\
\hline & & & & no exercise & $17 / 12 / 5$ & $62 \pm 6.7$ & NA \\
\hline \multirow[t]{2}{*}{ Whye 2011} & Singapore & Sedentary & 2 & unsupervised RE & $30 / 19 / 11$ & $57 \pm 7$ & $\begin{array}{l}65 \% \text { maximum heart rate; } 9 \text { resistive } \\
\text { exercises } 3 \text { sets of } 10 \text { repetitions }\end{array}$ \\
\hline & & & & unsupervised $A E$ & $30 / 22 / 8$ & $59 \pm 7$ & $\begin{array}{l}50 \text { min AT exercise; } 65 \% \text { maximum } \\
\text { heart rate }\end{array}$ \\
\hline \multirow[t]{2}{*}{ Morton 2012} & UK & NA & 1.65 & supervised AE & $27 / 6 / 21$ & $61 \pm 10$ & Walking in duration from 25 to $55 \mathrm{~min}$. \\
\hline & & & & no exercise & $27 / 6 / 21$ & $63 \pm 9$ & NA \\
\hline \multirow[t]{2}{*}{ Dede 2014} & Turkey & Sedentary & 3 & supervised AE & $30 / 15 / 15$ & $52.5 \pm 7.5$ & 60-75\% maximum heart rate \\
\hline & & & & no exercise & $30 / 16 / 14$ & $55.5 \pm 8.4$ & NA \\
\hline \multirow[t]{2}{*}{ BACCHI 2012} & Italy & NA & 4 & supervised AE & 20/6/14 & $57.2 \pm 1.6$ & 60-65\% maximum heart rate \\
\hline & & & & supervised RE & 20/6/14 & $55.6 \pm 1.7$ & $\begin{array}{l}30-50 \% 1 \mathrm{RM} \text {; three series of } \\
10 \text { repetitions }\end{array}$ \\
\hline \multirow[t]{2}{*}{ Ng 2010} & Singapore & Sedentary & 2 & supervised AE & $30 / 11 / 19$ & $57 \pm 7$ & NA \\
\hline & & & & supervised RE & $30 / 8 / 22$ & $59 \pm 7$ & $65 \% 1 \mathrm{RM} ;$ \\
\hline \multirow[t]{4}{*}{ Sparks 2013} & Netherlands & Sedentary & 9 & $\begin{array}{l}\text { flexibility } \\
\text { training }\end{array}$ & $10 / 8 / 2$ & $60.8 \pm 8.0$ & $65 \%$ maximum heart rate \\
\hline & & & & supervised AE & $12 / 6 / 6$ & $54.2 \pm 6.0$ & $50-80 \% \mathrm{VO}_{2}$ peak; \\
\hline & & & & supervised RE & 18/9/9 & $60.4 \pm 7.3$ & $\begin{array}{l}\text { Each session } 45-50 \text { min; each } \\
\text { set consisted of } 10 \text { to } 12 \text { repetitions }\end{array}$ \\
\hline & & & & combined & $12 / 6 / 6$ & $54.1 \pm 6.2$ & NA \\
\hline
\end{tabular}


Table 1 Characteristic of included studies (Continued)

\begin{tabular}{|c|c|c|c|c|c|c|c|}
\hline Author & Country & $\begin{array}{l}\text { Type of } \\
\text { patients }\end{array}$ & $\begin{array}{l}\text { Study } \\
\text { period } \\
\text { (months) }\end{array}$ & Interventions & $\begin{array}{l}\text { Sample } \\
\text { N/F/M }\end{array}$ & $\begin{array}{l}\text { Mean age } \\
\text { (years) }\end{array}$ & Exercise prescription \\
\hline \multirow[t]{4}{*}{ Gavin 2010} & \multirow[t]{4}{*}{ Canada } & \multirow[t]{4}{*}{ Sedentary } & \multirow[t]{4}{*}{6} & no exercise & $63 / 22 / 41$ & $54.8 \pm 7.2$ & NA \\
\hline & & & & supervised $A E$ & $60 / 21 / 39$ & $53.9 \pm 6.6$ & $65 \%$ maximum heart rate; $50 \%$ of $\mathrm{VO}_{2}$ peak \\
\hline & & & & supervised RE & $64 / 24 / 40$ & $54.7 \pm 7.5$ & 2 to 3 times weekly; maximum of 8 repetitions \\
\hline & & & & combined & $64 / 24 / 40$ & $53.5 \pm 7.3$ & $\begin{array}{l}\text { full Aerobic program plus the full } \\
\text { Resistance program }\end{array}$ \\
\hline \multirow[t]{2}{*}{ Madden 2009} & \multirow[t]{2}{*}{ Canada } & \multirow[t]{2}{*}{ NA } & \multirow[t]{2}{*}{3} & supervised AE & $18 /-/-$ & $71.7 \pm 1.1$ & $\begin{array}{l}\text { Each session } 60 \mathrm{~min} \text {; } 60-75 \% \text { maximum } \\
\text { heart rate }\end{array}$ \\
\hline & & & & An-AE & $18 /-/-$ & $71.1 \pm 0.9$ & $\begin{array}{l}3 \text { times weekly; no aerobic component } \\
\text { and consisted of nonaerobic core and } \\
\text { dumbbells exercises }\end{array}$ \\
\hline \multirow[t]{2}{*}{ Madden 2011} & \multirow[t]{2}{*}{ Canada } & \multirow[t]{2}{*}{ Sedentary } & \multirow[t]{2}{*}{3} & supervised AE & $21 / 15 / 25$ & $71.9 \pm 1.1$ & 3 times weekly; each session 60 min \\
\hline & & & & $A n-A E$ & & $71.3 \pm 0.9$ & $\begin{array}{l}3 \text { times weekly; no aerobic component } \\
\text { and consisted of nonaerobic core and } \\
\text { dumbbells exercises }\end{array}$ \\
\hline \multirow[t]{2}{*}{ William 2011} & \multirow{2}{*}{$\begin{array}{l}\text { New } \\
\text { Zealand }\end{array}$} & \multirow[t]{2}{*}{ NA } & \multirow[t]{2}{*}{4} & supervised RE & $9 / 6 / 3$ & $48 \pm 6$ & two to three sets of eight major exercises \\
\hline & & & & supervised AE & $9 / 7 / 2$ & $51 \pm 4$ & 3 times weekly; each session 40-60 min; \\
\hline \multirow{2}{*}{$\begin{array}{l}\text { Kadoglou } \\
2014\end{array}$} & \multirow[t]{2}{*}{ Greece } & \multirow[t]{2}{*}{ Sedentary } & \multirow[t]{2}{*}{6} & supervised $A E$ & $30 / 17 / 13$ & $59.33 \pm 4.76$ & $50-75 \%, \mathrm{VO}_{2}$ peak; each session $60 \mathrm{~min}$ \\
\hline & & & & no exercise & $30 / 18 / 12$ & $63.82 \pm 7.03$ & NA \\
\hline \multirow[t]{2}{*}{ ALAM 2004} & \multirow[t]{2}{*}{ UK } & \multirow[t]{2}{*}{ NA } & \multirow[t]{2}{*}{6} & supervised AE & $9 / 5 / 4$ & $59.5 \pm 2.5$ & $60-85 \%, \mathrm{VO}_{2}$ peak; supervised by trainer \\
\hline & & & & unsupervised $\mathrm{AE}$ & $9 / 4 / 5$ & $55.3 \pm 23.2$ & NA \\
\hline \multirow[t]{2}{*}{ Tessier 2000} & Canada & NA & 3 & supervised AE & 19/7/12 & $69.3 \pm 4.2$ & $60-79 \%$ maximum heart rate \\
\hline & & & & no exercise & 20/9/11 & $69.5 \pm 5.1$ & NA \\
\hline Church 2011 & American & Sedentary & 3 & no exercise & $41 / 28 / 13$ & $58.6 \pm 8.2$ & NA \\
\hline & & & & supervised RE & $73 / 43 / 30$ & $56.9 \pm 8.7$ & $\begin{array}{l}3 \text { times per week; each set consisted } \\
\text { of } 10 \text { to } 12 \text { repetitions }\end{array}$ \\
\hline & & & & supervised AE & $72 / 45 / 27$ & $53.7 \pm 9.1$ & $\begin{array}{l}50-80 \% \text { maximum heart rate; } \\
150 \text { min per week }\end{array}$ \\
\hline & & & & Combined & $76 / 49 / 27$ & $55.4 \pm 8.3$ & NA \\
\hline Kwon 2010b & Korea & NA & 6 & unsupervised AE & $13 / 13 / 0$ & $55.5 \pm 7.5$ & 5 times per week; \\
\hline & & & & no exercise & $14 / 14 / 0$ & $57.5 \pm 8.6$ & NA \\
\hline Winnick 2008 & USA & NA & 4 & unsupervised $\mathrm{AE}$ & $\begin{array}{l}15 \text { (the } \\
\text { white) }\end{array}$ & $49.5 \pm 2.9$ & $\begin{array}{l}10 \text { repetition performed on } \\
\text { each of eight machines }\end{array}$ \\
\hline & & & & unsupervised RE & $\begin{array}{l}18 \text { (the } \\
\text { white) }\end{array}$ & $50.3 \pm 3.5$ & 3 times weekly; each session 30-40mijn \\
\hline & & & & unsupervised $\mathrm{AE}$ & 24 (African) & $50.7 \pm 2.0$ & NA \\
\hline & & & & unsupervised RT & 12 (African) & $46.2 \pm 2.0$ & NA \\
\hline
\end{tabular}

NOTE: AE: aerobic exercise; RE: resistance exercise; NA: not available; RM: repetition maximum

HbA1c (Table 2, Additional file 1: Appendix 6). Compared to unsupervised aerobic and unsupervised resistance exercises, supervised aerobic $(-0.60,95 \% \mathrm{CI}:-0.83 \%$ to $0.30 \%$; $-0.60,95 \% \mathrm{CI}:-0.83 \%$ to $-0.20 \%$; respectively) and supervised resistance $(-0.53 \%$ lower, $95 \% \mathrm{CI}:-0.75 \%$ to $0.30 \%$; -0.53 , $95 \%$ CI: $-0.83 \%$ to $-0.23 \%$; respectively) exercises showed more benefit in reducing HbA1c (Table 2, Additional file 1: Appendix 6). Combined exercise resulted in the most significant reduction in HbA1c when compared with supervised aerobic $(-0.23,95 \% \mathrm{CI}:-0.30 \%$ to $0.08 \%)$, unsupervised aerobic $(-0.75,95 \%$ CI: $-0.98 \%$ to -
$0.53 \%)$, supervised resistance $(-0.23,95 \% \mathrm{CI}:-0.38 \%$ to $0.15 \%)$, and unsupervised resistance $(-0.75,95 \% \mathrm{CI}$ : $-0.98 \%$ to $-0.45 \%$ ) exercises (Table 2, Additional file 1: Appendix 6). Furthermore, combined exercise also showed the greatest potential as the best intervention to improve HbA1c (P-score $=0.99$, Additional file 1: Appendix 6). However, there were no significant differences between the effectiveness of the other exercises in reducing HbA1c levels (Table 2).

Subgroup analysis (Additional file 1: Appendix 5) showed that supervised aerobic and supervised resistance 


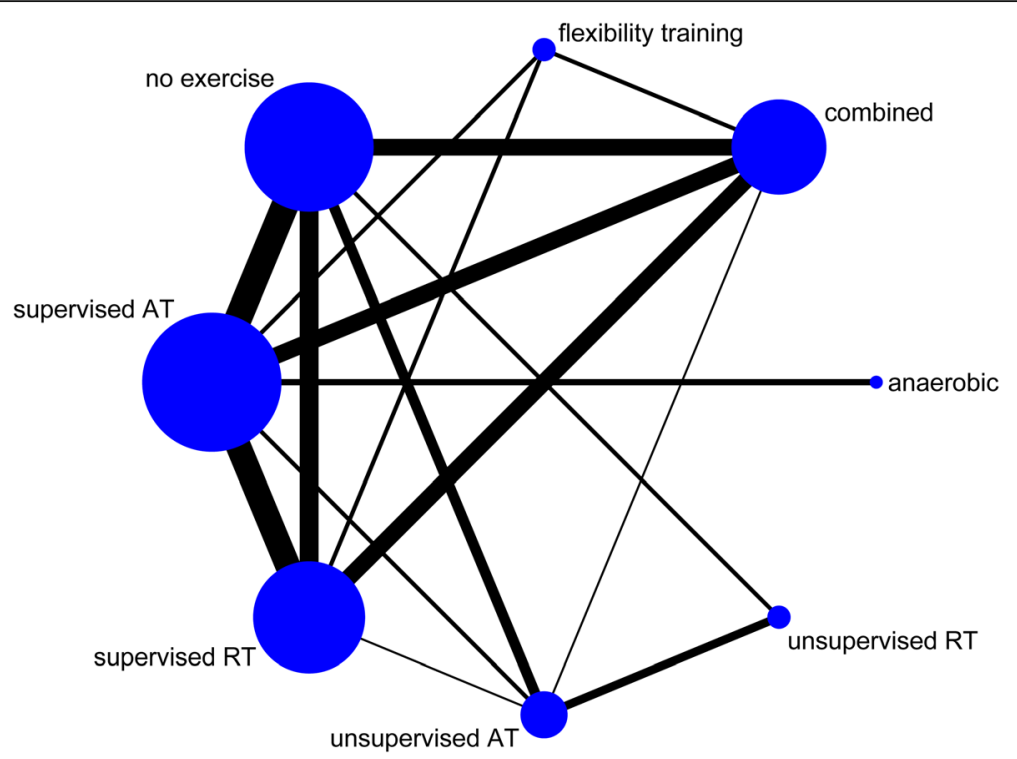

Fig. 2 network plot

forms of exercise were not significantly better than unsupervised aerobic exercise in reducing HbA1c levels when the study duration was less than 6 months $\left(P_{\text {interac- }}\right.$ tion $<0.05)$. Significant interaction discrepancies were not found in our analyses of other subgroups.

Similar to the effects on $\mathrm{HbA1c}$, supervised aerobic significantly reduced FBG by $9.38 \mathrm{mg} / \mathrm{dl}$ (Table 2, Additional file 1: Appendix 4), and ranking probability showed that supervised aerobic exercise had the most significant ability to reduce FBG (P-score $=0.82$, Additional file 1: Appendix 6). Subgroup analyses did not show significant interaction discrepancy between any of the subgroup factors (Additional file 1: Appendix 5).

\section{Weight loss}

Seventeen studies involving 662 patients reported weight loss and were included in our network meta-analysis.

Compared to unsupervised aerobic exercise, combined $(-8.37 \mathrm{~kg}, 95 \% \mathrm{CI}$ : $-13.39 \mathrm{~kg}$ to $-3.35 \mathrm{~kg})$, supervised aerobic $(-5.02 \mathrm{~kg}, 95 \% \mathrm{CI}$ : $-8.37 \mathrm{~kg}$ to $-1.67 \mathrm{~kg})$, supervised resistance $(-5.02 \mathrm{~kg}, 95 \% \mathrm{CI}:-9.21 \mathrm{~kg}$ to $-0.84 \mathrm{~kg})$, and anaerobic $(-8.37 \mathrm{~kg}, 95 \% \mathrm{CI}:-15.07 \mathrm{~kg}$ to $-1.67 \mathrm{~kg}$ ) forms of exercise showed greater weight reduction (Table 2, Additional file 1: Appendix 6). Subgroup analysis showed that these differences tended to be greater in studies of longer duration (Additional file 1: Appendix 5).

Combined exercise also showed more benefit in terms of weight loss compared to the effects of no exercise $(-5.02 \mathrm{~kg}, 95 \% \mathrm{CI}:-9.21 \mathrm{~kg}$ to $-0.84 \mathrm{~kg})$. In addition, combined exercise showed the most significant effectiveness in terms of weight loss $(P$-score $=$ 0.86, Additional file 1: Appendix 6).

\section{Cardiovascular risk factors}

Twenty-two studies involving 1323 patients reported SBP, DBP, TC, TG, LDL and HDL. Of these, one study [68] was not included in our network meta-analysis of TC due to imbalance in the baseline.

Compared to no exercise, supervised aerobic (TC: $20.24 \mathrm{mg} / \mathrm{dl}, 95 \% \mathrm{CI}:-27.60 \mathrm{mg} / \mathrm{dl}$ to $-11.04 \mathrm{mg} / \mathrm{dl}$; TG: $-19.34 \mathrm{mg} / \mathrm{dl}, 95 \% \mathrm{CI}:-29.76 \mathrm{mg} / \mathrm{dl}$ to $-5.95 \mathrm{mg} /$ $\mathrm{dl}$; LDL: $-11.88 \mathrm{mg} / \mathrm{dl}$, 95\%CI: $-21.60 \mathrm{mg} / \mathrm{dl}$ to $1.08 \mathrm{mg} / \mathrm{dl}$; HDL: $-3.66 \mathrm{mg} / \mathrm{dl}$, 95\%CI: $-5.04 \mathrm{mg} / \mathrm{dl}$ to $-1.83 \mathrm{mg} / \mathrm{dl}$ ), supervised resistance (SBP: $-5.20 \mathrm{mmHg}$, 95\%CI: $-9.10 \mathrm{mmHg}$ to $-1.30 \mathrm{mmHg}$;C: $-22.08 \mathrm{mg} / \mathrm{dl}$, 95\%CI: $-31.28 \mathrm{mg} / \mathrm{dl}$ to $-11.04 \mathrm{mg} / \mathrm{dl}$; TG: $-16.37 \mathrm{mg} / \mathrm{dl}$, 95\%CI: $-28.27 \mathrm{mg} / \mathrm{dl}$ to $-4.46 \mathrm{mg} / \mathrm{dl}$; HDL: $-4.58 \mathrm{mg} / \mathrm{dl}$, 95\%CI: $-6.87 \mathrm{mg} / \mathrm{dl}$ to $2.75 \mathrm{mg} / \mathrm{dl}$ ) and combined (TG: $37.20 \mathrm{mg} / \mathrm{dl}$, 95\%CI: $-49.10 \mathrm{mg} / \mathrm{dl}$ to $-23.81 \mathrm{mg} / \mathrm{dl}$ ) exercises showed better improvement in SBP, TC, TG and LDL (Table 2, Additional file 1: Appendix 6).

Supervised aerobic (-23.92 mg/dl, 95\%CI: $-33.12 \mathrm{mg} /$ $\mathrm{dl}$ to $-12.88 \mathrm{mg} / \mathrm{dl}$ ) and supervised resistance ($25.76 \mathrm{mg} / \mathrm{dl}, 95 \% \mathrm{CI}:-36.80 \mathrm{mg} / \mathrm{dl}$ to $-14.72 \mathrm{mg} / \mathrm{dl})$ exercise showed greater improvement in TC and HDL compared with the effects of combined exercise, while combined exercise induced a greater reduction in TG than supervised aerobic $(-25.76 \mathrm{mg} / \mathrm{dl}, 95 \% \mathrm{CI}$ : $-46.00 \mathrm{mg} / \mathrm{dl}$ to $-3.68 \mathrm{mg} / \mathrm{dl}$ ) and supervised resistance (- 29.44 mg/dl, 95\%CI: $-47.84 \mathrm{mg} / \mathrm{dl}$ to $-7.36 \mathrm{mg} / \mathrm{dl}) \mathrm{ex}-$ ercises (Table 2).

Supervised resistance showed the most significant improvements in HDL, LDL and TC $(P$-score $=0.74,0.79$, 0.92, respectively, Additional file 1: Appendix 6), and combined exercise showed the most significant improvements in TG (P-score $=0.99)$. 


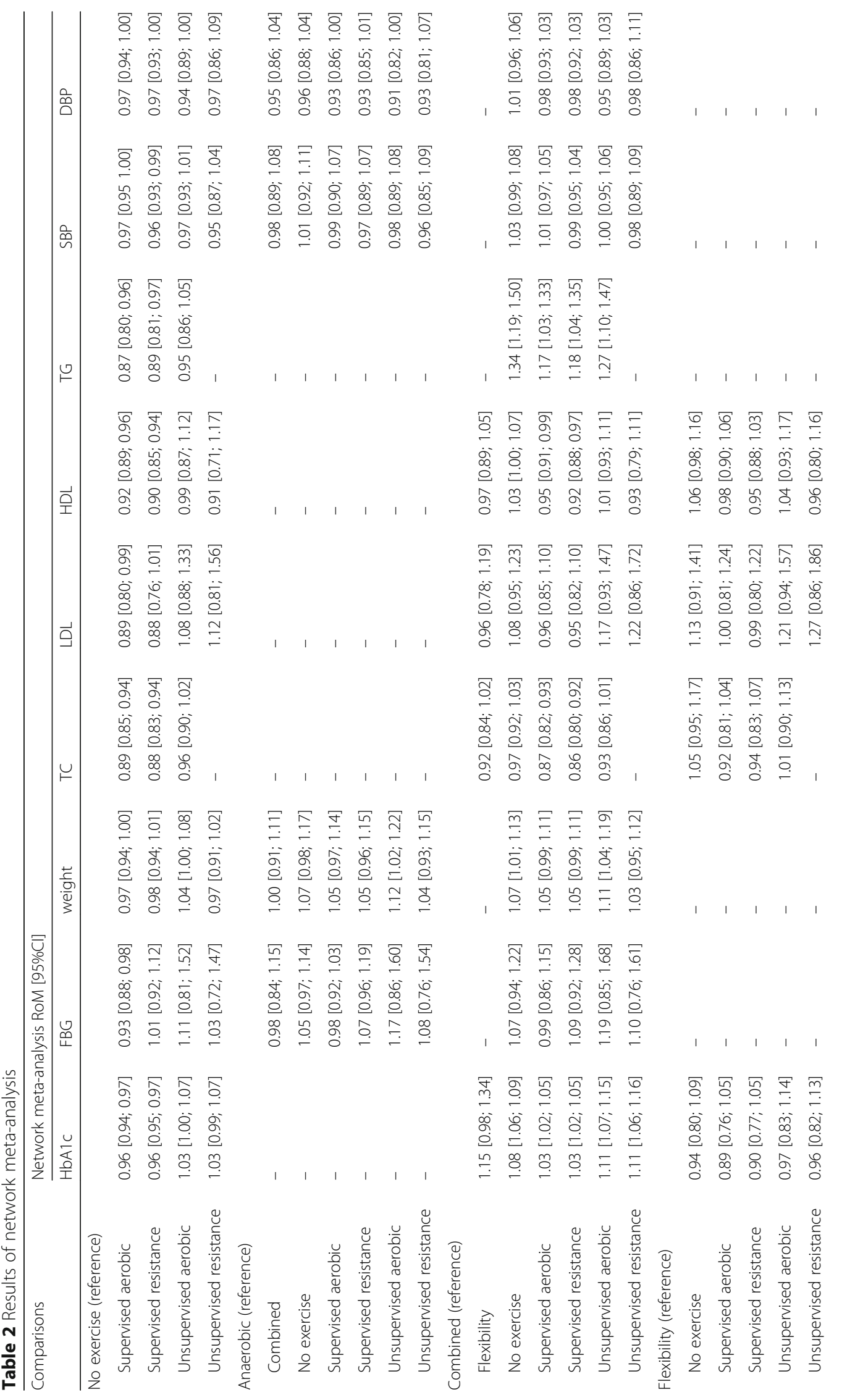




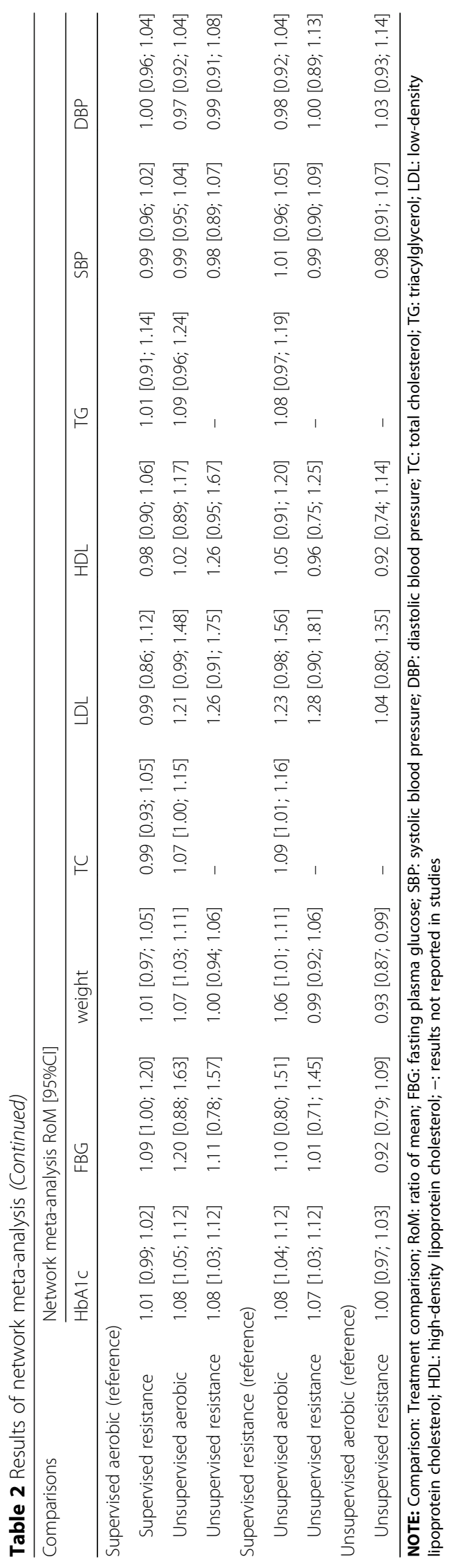


Subgroup analyses showed that supervised aerobic exercise was associated with a greater reduction in SBP than unsupervised aerobic exercise in the older population $\left(P_{\text {interaction }}<0.05\right)$, a greater reduction in LDL than combined exercise in the older population $\left(P_{\text {interaction }}<\right.$ $0.05)$, and a greater reduction in HDL than supervised resistance exercise with longer disease duration $\left(P_{\text {interac- }}\right.$ tion < 0.05) (Additional file 1: Appendix 5).

\section{Inconsistency between direct and indirect comparisons}

Assessment of inconsistency between direct and indirect comparisons using a node-splitting model showed that there were no inconsistencies among most studies $(P>0.05)$ (Additional file 1: Appendix 4).

\section{Discussion}

In our study, we used both direct and indirect evidence to evaluate the relative effects of different exercises on glycemic control, cardiovascular risk factors, and weight loss in patients with T2DM. In particular, we separated the differences between supervised and unsupervised forms of exercise. Integrating the currently available data, our network meta-analysis indicated that combined exercise was as effective in reducing $\mathrm{HbA} 1 \mathrm{c}$ as supervised aerobic and supervised resistance forms of exercise. Supervised aerobic training and supervised resistance training were more powerful in improving SBP, TC, and HDL than combined exercise. P-Score ranking revealed that combined, supervised aerobic, and supervised resistance forms as the top three exercise modalities. Moreover, supervised exercise showed more benefit than unsupervised exercise for most comparison groups.

Our study suggested those who wished to improve their HbA1c through lifestyle management to engage in combined exercise. Recently, increasing importance has been attached to the effect of combined exercise. One study [49] showed that combined exercise was more effective than either aerobic or resistance training alone in reducing $\mathrm{HbA1c}$, which was consistent with the findings of our analysis. Therefore, Appropriate exercise is recommended for T2DM patients as a part of their therapy, although this approach is usually unsuccessful unless the training is supervised. It could be speculated that this was because therapeutic exercise strategies that were designed by professional trainers were often ineffective in improving the adverse lipid profile and decreasing insulin resistance. Evidence from RCTs showed a decrease in $\mathrm{HbA1c}$, fasting insulin concentration, and FBG in the supervised aerobic group, but not in the corresponding unsupervised group [63]. The results of our study also showed that supervised aerobic/resistance could effectively manage patients with T2DM than unsupervised group.
Onset of T2DM, cardiovascular disease and even cardiovascular mortality are inversely related to cardiorespiratory fitness [71]. Compared with the non-diabetic population, the population with T2DM is associated with a higher risk of cardiovascular disease (2 to 4-fold increase) [72]. Regular aerobic exercise can increase insulin sensitivity, which improves the adverse lipid profile $[73,74]$. It has been shown that resistance exercise can benefit all adults and patients with T2DM by improving physical function, fat mass, lipid profiles, cardiovascular health, blood pressure, and insulin sensitivity $[75,76]$. Meta-analyses performed by Kelley [77] showed that aerobic exercise was more efficient for lowering LDL in patients with T2DM than other types of training. However, the meta-analyses by Kelley failed to show the comparative effectiveness of aerobic exercise for improving other cardiovascular risk factors. Our meta-analysis showed that both supervised aerobic and supervised resistance exercise had the similar effect in improving LDL and TC. Thus, it may be significantly helpful for clinicians, policy makers, and patients with T2DM to recommend either form of exercise for the prevention of cardiovascular disease.

Schwingshackl [32] compared the effects of different training modalities on glycemic control and blood lipids in patients with T2DM. The objective of their study was similar to that of ours. However, we conducted a more comprehensive analysis in that we included not only supervised aerobic exercise and supervised resistance exercise, but also unsupervised aerobic and unsupervised resistance exercise, as well as no exercise, and flexibility training. In addition, we focused on more outcomes including glycemic control, cardiovascular risk factors, and weight loss. Importantly, because the different units of measurement for all outcomes of interest were used in included RCTs, we usually analyzed a pool of these studies using SMD. However, since it is difficult to explain this parameter to evidence users, we employed the RoM to measure the relative effect differences between the intervention and control groups. Finally we calculated absolute effect differences using no exercise as the baseline risk, which is more straightforward and more understandable to evidence users.

To evaluate the evidence in a specific population, we performed four subgroup analyses in term of study duration, diabetes duration, age, and type of population. We found that supervised aerobic exercise and supervised resistance exercise showed more benefit in reducing HbA1c than unsupervised aerobic exercise only when the study duration was longer than 6 months. Supervised aerobic exercise showed a greater reduction in SBP than unsupervised aerobic exercise in the older population, and a greater reduction in HDL than supervised resistance for patients with longer disease duration. No 
significant interaction discrepancies in most of the outcomes were found for most of the comparison groups.

Some limitations of our study should be noted. First, we used RoM (post-intervention/post-control) to account for the changes from baseline and some RCTs showed significant differences in the baseline; therefore, those RCTs were not included in our final network meta-analysis model. Second, previous dose-response meta-regression analysis [78] revealed that the reduction in HbA1c was associated with exercise frequency for supervised aerobic exercise, and associated with weekly volume of resistance for supervised combined exercise although further studies are required to confirm these associations. Finally, we planned to include quality of life as a primary outcome in our study protocol; however, the scales for measurement of quality of life were widely inconsistent. For example, Holton's study [79] used a 36-item Short-Form Health Survey, while Bello's study [80] used the WHO Quality of Life questionnaire, and Fritz's study [81] used the Swedish Health-Related Quality of Life questionnaire. Our network meta-analysis included eight interventions and nine outcomes with extremely complex networks; therefore, we consider that quality of life should be evaluated in a separate study.

\section{Conclusions}

Combined exercise showed more pronounced improvement in HbA1c than either supervised aerobic exercise or supervised resistance exercise alone; however, the decrease in some cardiovascular risk factors was less marked. In terms of weight loss, there were no significant differences among the combined, supervised aerobic and supervised resistance forms of exercise.

\section{Additional file}

Additional file 1: Appendix 1 Search strategies. Appendix 2 Definition of interventions. Appendix 3 Results of risk of bias. Appendix 4 Results of direct, indirect, network meta-analyses, and inconsistency. Appendix 5 subgroup analyses. Appendix 6 Absolute effect estimates of different exercise modalities using no exercise as baseline risk (PDF $400 \mathrm{~kb}$ )

\section{Abbreviations}

ACSM: American College of Sports Medicine; ADA: American Diabetes Association; BPTA: Belgian Physical Therapy Association; combined exercise: combined aerobic exercise and resistance exercise; DBP: diastolic blood pressure; ESC: European Society of Cardiology; ESSA: Exercise and Sports Science Australia; FBG: fasting plasma glucose; HDL: high-density lipoprotein cholesterol; LDL: low-density lipoprotein cholesterol; MD: mean difference; RCT: randomized controlled trials; RoM: ratio of mean; SBP: systolic blood pressure; SMD: standardized mean difference; SNIPH: Swedish National Institute of Public Health; T2DM: Type 2 diabetes; TC: total cholesterol; TG: triacylglycerol

\section{Acknowledgements}

We thank all members of our study team for their whole-hearted cooperation and the original authors of the included studies for their wonderful work. We also thank Evidence-Based Medicine Center of Lanzhou University for methodological support.

\section{Available of data and materials}

The datasets and any other materials of our study are available from corresponding author on request.

\section{Competing interests}

None.

\section{Funding}

None.

\section{Authors' contributions}

$L G, J H T, G W D$ and $K H Y$ planed and designed the research; $L G$ and JHT provided methodological support/advice; BP and CYG tested the feasibility of the study; BP, CYG, YJC, XH, LQZ, YQX, and HQS extract data; BP and LG performed the statistical analysis; BP wrote the manuscript; all authors approved the final version of the manuscript.

Ethics approval and consent to participate

Ethics approval and participants consent are not required because this study is a meta-analysis based on the published studies.

\section{Consent for publication}

Not applicable.

\section{Publisher's Note}

Springer Nature remains neutral with regard to jurisdictional claims in published maps and institutional affiliations.

\section{Author details}

'Department of Social Medicine and Health Management, School of Public Health, Lanzhou University, Lanzhou 730000, China. ${ }^{2}$ The First Clinical Medical College, Lanzhou University, Lanzhou 730000, China.

${ }^{3}$ Evidence-Based Medicine Center, School of Basic Medical Sciences, Lanzhou University, No. 199, Dong gang West Road, Chengguan District, Lanzhou City, Gansu province, China.

Received: 22 January 2018 Accepted: 17 July 2018

Published online: 25 July 2018

\section{References}

1. International Diabetes Federation. IDF diabetes atlas. 6th edn. Brussels International Diabetes Federation, 2013.

2. Shaw JE, Sicree RA, Zimmet PZ. Global estimates of the prevalence of diabetes for 2010 and 2030. Diabetes Res Clin Pract. 2010:87:4-14.

3. Sullivan PW, Morrato EH, Ghushchyan V, Wyatt HR, Hill JO. Obesity, inactivity, and the prevalence of diabetes and diabetes-related cardiovascular comorbidities in the U.S., 2000-2002. Diabetes Care. 2005;28:1599-603.

4. American Diabetes Association. Standards of medi in diabetes-2012. Diabetes Care. 2012; 35 Suppl: 11-63.

5. Sudeck G, Honer O. Volitional interventions within cardiac exercise therapy (VIN-CET): long-term effects on physical activity and health-related quality of life. Applied Psychology-Health and Well Being. 2011:3:151-71.

6. Eckel RH, Grundy SM, Zimmet PZ. The metabolic syndrome. Lancet. 2005; 365:1415-28.

7. UK Prospective Diabetes Study Group. Tight blood pressure control and risk of macrovascular and microvascular complications in type 2 diabetes: UKPDS 38. BMJ. 1998;317:703-13.

8. Wandell PE. Quality of life of patients with diabetes mellitus-an overview of research in primary health cares in the Nordic countries. Scand J Prim Health Care. 2005;23:68-74

9. Ruiz JR, Sui X, Lobelo F, Morrow JRJ, Jackson AW, Sjöström M, et al. Association between muscular strength and mortality in men: prospective cohort study. BMJ. 2008:337:a439.

10. Wei M, Gibbons LW, Kampert JB, Nichaman MZ, Blair SN. Low cardiorespiratory fitness and physical inactivity as predictors of mortality in men with type 2 diabetes. Ann Intern Med. 2000:132:605-11.

11. Church TS, Cheng YJ, Earnest CP, Barlow CE, Gibbons LW, Priest EL, et al. Exercise capacity and body composition as predictors of mortality among men with diabetes. Diabetes Care. 2004;27:83-8.

12. Castaneda C, Layne JE, Munoz-Orians L, Gordon PL, Walsmith J, Foldvari M, et al. A randomized controlled trial of resistance exercise training to 
improve glycemic control in older adults with type 2 diabetes. Diabetes Care. 2002;25:2335-41.

13. Mourier A, Gautier JF, De Kerviler E, Bigard AX, Villette JM, Garnier JP, et al. Mobilization of visceral adipose tissue related to the improvement in insulin sensitivity in response to physical training in NIDDM. Effects of branchedchain amino acid supplements. Diabetes Care. 1997;20:385-91.

14. Toledo FG, Menshikova EV, Ritov VB, Azuma K, Radikova Z, DeLany J, et al. Effects of physical activity and weight loss on skeletal muscle mitochondria and relationship with glucose control in type 2 diabetes. Diabetes. 2007;56: 2142-7.

15. Mendes R, Sousa N, Almeida A, Subtil P, Guedes-Marques F, Reis VM, et al. Exercise prescription for patients with type 2 diabetes-a synthesis of international recommendations: narrative review. Br J Sports Med. 2016;22:1379-81.

16. Ryden L, Grant PJ, Anker SD, et al. ESC guidelines on diabetes, pre-diabetes, and cardiovascular diseases developed in collaboration with the EASD: the task force on diabetes, pre-diabetes, and cardiovascular diseases of the European Society of Cardiology (ESC) and developed in collaboration with the European Association for the Study of diabetes (EASD). Eur Heart J. 2013:34:3035-87.

17. Colberg SR, Sigal RJ, Fernhall B, et al. Exercise and type 2 diabetes: the American College of Sports Medicine and the American Diabetes Association: joint position statement. Diabetes Care. 2010;33:e147-67.

18. Hansen D, Peeters S, Zwaenepoel B, et al. Exercise assessment and prescription in patients with type 2 diabetes in the private and home care setting: clinical recommendations from AXXON (Belgian physical therapy association). Phys Ther. 2013;93:597-610.

19. Hordern MD, Dunstan DW, Prins JB, et al. Exercise prescription for patients with type 2 diabetes and pre-diabetes: a position statement from exercise and sport science Australia. J Sci Med Sport. 2012;15:25-31.

20. Diabetes Canada Clinical Practice Guidelines Expert Committee, Sigal RJ, Armstrong MJ, Bacon SL, Boulé NG, Dasgupta K, et al. Physical Activity and Diabetes. Can J Diabetes. 2018; 42 Suppl 1: 54-63.

21. Baldi JC, Snowling N. Resistance training improves glycaemic control in obese type 2 diabetic men. Int J Sports Med. 2003;24:419-23.

22. Dunstan DW. Aerobic exercise and resistance training for the management of type 2 diabetes mellitus. Nat Clin Pract Endocrinol Metab. 2008:4:250-1.

23. Hovanec N, Sawant A, Overend TJ. Resistance training and older adults with type 2 diabetes. J Aging Res. 2012;2012:284635.

24. Cai H, Li G, Zhang P, Xu D, Chen L. Effect of exercise on the quality of life in type 2 diabetes mellitus: a systematic review. Qual Life Res. 2017;26:515-30.

25. Bafeta A, Trinquart L, Seror R, Ravaud P. Reporting of results from network meta-analyses: methodological systematic review. BMJ. 2014;348:g1741.

26. American Diabetes Association. 2. Classification and Diagnosis of Diabetes. Diabetes Care. 2017; 40 Suppl: 11-24.

27. Higgins JPT, Green S. Cochrance Handbook for Systematic Reviews of Interventions Version 5.1.0 [EB/OL]. The Cochrane Collaboration. 2011. http://www.cochrane-handbook.org. Accessed 27 May 2017.

28. Rücker G, Schwarzer G, Krahn U. netmeta: Network meta-analysis using Frequentist methods. https://cran.r-project.org/web/packages/netmeta/ netmeta.pdf. Accessed 28 May 2017.

29. Rücker G, Schwarzer G. Ranking treatments in frequentist network meta-analysis works without resampling methods. BMC Med Res Methodol. 2015:15:58.

30. Brehm BJ, Lattin BL, Summer SS, Boback JA, Gilchrist GM, Jandacek RJ, D'Alessio DA. One-year comparison of a high-monounsaturated fat diet with a high-carbohydrate diet in type 2 diabetes. Diabetes Care. 2009;32:215-20.

31. Friedrich JO, Adhikari N, Herridge MS, Beyene J. Meta-analysis: low-dose dopamine increases urine output but does not prevent renal dysfunction or death. Ann Intern Med. 2005;142:510-24.

32. SchwingshackI L, Missbach B, Dias S, König J, Hoffmann G. Impact of different training modalities on glycaemic control and blood lipids in patients with type 2 diabetes: a systematic review and network metaanalysis. Diabetologia. 2014;57:1789-97.

33. World Health Organization. Indicators for the Minimum Data Set Project on Ageing: A Critical Review in sub-Saharan Africa. http://www.who.int/ healthinfo/survey/ageing_mds_report_en_daressalaam.pdf. 2001. Accessed 25 May 2018.

34. Dunstan DW, Daly RM, Owen N, Jolley D, Vulikh E, Shaw J, Zimmet P. Home-based resistance training is not sufficient to maintain improved glycemic control following supervised training in older individuals with type 2 diabetes. Diabetes Care. 2005;28:3-9.
35. Cheung NW, Cinnadaio N, Russo M, Marek S. A pilot randomised controlled trial of resistance exercise bands in the management of sedentary subjects with type 2 diabetes. Diabetes Res Clin Pract. 2009;83:e68-71.

36. Franciele R. Figueira FR, Umpierre D, Casali KR. Aerobic and combined exercise sessions reduce glucose variability in type 2diabetes: crossover randomized trial. PLoS One. 2013:8: e57733.

37. Larose J, Sigal RJ, Khandwala F, Prud'homme D, Boulé NG, Kenny GP. Diabetes aerobic and resistance exercise (DARE) trial investigators. Associations between physical fitness and $\mathrm{HbA}_{1}(\mathrm{c})$ in type 2 diabetes mellitus. Diabetologia. 2011;54:93-102.

38. Stolinski M, Alam S, Jackson NC, Shojaee-Moradie F, Pentecost C, Jefferson W, Christ ER, Jones RH, Umpleby AM. Effect of 6-month supervised exercise on low-density lipoprotein apolipoprotein B kinetics in patients with type 2 diabetes mellitus. Metabolism. 2008:57:1608-14.

39. Cindy Li, Whye NG, Tai ES, Goh SY, Wee H. Health status of older adults with Type 2 diabetes mellitus after aerobic or resistance training: A randomised trial. Health Qual Life Outcomes. 2011; 9:59.

40. Arslan M, Ipekci SH, Kebapcilar L. Int Sch Res Notices. 2014; https://doi.org/ 10.1155/2014/820387. Accessed 30 May 2017

41. Yavari A, Hajiyev AM, Naghizadeh F. The effect of aerobic exercise on glycosylated hemoglobin values in type 2 diabetes patients. J Sports Med Phys Fitness. 2010;50:501-5.

42. Okada S, Hiuge A, Makino H, Nagumo A, Takaki H, Konishi H, et al. Effect of exercise intervention on endothelial function and incidence of cardiovascular disease in patients with type 2 diabetes. J Atheroscler Thromb. 2010;31(17):828-33.

43. Shenoy S, Guglani R, Sandhu JS. Effectiveness of an aerobic walking program using heart rate monitor and pedometer on the parameters of diabetes control in Asian Indians with type 2 diabetes. Prim Care Diabetes. 2010;4:41-5.

44. Belli T, Ribeiro LF, Ackermann MA, Baldissera V, Gobatto CA, Galdino da Silva R. Effects of 12-week overground walking training at ventilatory threshold velocity in type 2 diabetic women. Diabetes Res Clin Pract. 2011;93:337-43.

45. Choi KM, Han KA, Ahn HJ, Hwang SY, Hong HC, Choi HY, et al. Effects of exercise on sRAGE levels and Cardiometabolic risk factors in patients with type 2 diabetes: a randomized controlled trial. J Clin Endocrinol Metab. 2012;97:3751-8.

46. Church TS, Blair SN, Cocreham S, Johannsen N, Johnson W, Kramer K, et al. Effects of aerobic and resistance training on hemoglobin A1C levels in patients with type 2 diabetes: a randomized controlled trial. JAMA. 2010; 24(304):2253-62.

47. Kwon HR, Min KW, Ahn HJ, et al. Effects of aerobic exercise on abdominal fat, Tigh muscle mass and muscle strength in type 2 diabetic subject. Korean Diabetes J. 2010;34:23-31.

48. Reid RD, Tulloch HE, Sigal RJ, Kenny GP, Fortier M, McDonnell L, Wells GA, Boulé NG, Phillips P, Coyle D. Effects of aerobic exercise, resistance exercise or both, on patient-reported health status and well-being in type 2 diabetes mellitus. Diabetologia. 2010;53:632-40.

49. Sigal RJ, Kenny GP, Boulé NG, Wells GA, Prud'homme D, Fortier M, Reid RD, Tulloch $H$, Coyle D, Phillips $P$, Jennings $A$, Jaffey J. Effects of aerobic training, resistance training, or both on glycemic control in type 2 diabetes. Ann Intern Med. 2007;18(147):357-69.

50. Arora E, Shenoy S, Sandhu JS. Effects of resistance training on metabolic prof le of adults with type 2 diabetes. Indian J Med Res. 2009;129:515-9.

51. Morton RD, West DJ, Stephens JW, Bain SC, Bracken RM. Heart rate prescribed walking training improves cardiorespiratory fitness but not glycaemic control in people with type 2 diabetes. J Sports Sci. 2010;28:93-9.

52. Nesrin Doğan Dede, Süleyman Hilmi Ipekci, Levent Kebapcilar. Influence of Exercise on Leptin, Adiponectin and Quality of Life in Type 2 Diabetics. Turk Jem. 2015; 19: 7-13.

53. Bacchi E, Negri C, Zanolin ME, et al. Metabolic effects of aerobic training and resistance training in type 2 diabetic subjects. Diabetes Care. 2012;35:676-82.

54. Ng CL, Goh SY, Malhotra R, Ostbye T, Tai ES. Minimal difference between aerobic and progressive resistance exercise on metabolic profile and fitness in older adults with diabetes mellitus: a randomised trial. J Physiother. 2010; 56:163-70.

55. Sparks LM, Johannsen NM, Church TS, Earnest CP, Moonen-Kornips E, Moro C, Hesselink MK, Smith SR, Schrauwen P. Nine months of combined training improves ex vivo skeletal muscle metabolism in individuals with type 2 diabetes. J Clin Endocrinol Metab. 2013;98:1694-702. 
56. Gavin C, Sigal RJ, Cousins M, Menard ML, Atkinson M, Khandwala F, Kenny GP, Proctor S, Ooi TC; Diabetes Aerobic and Resistance Exercise (DARE) trial investigators. Resistance exercise but not aerobic exercise lowers remnantlike lipoprotein particle cholesterol in type 2 diabetes: A randomized controlled trial. Atherosclerosis. 2010; 213:552-7.

57. Ku YH, Han KA, Ahn H, Kwon H, Koo BK, Kim HC, Min KW. Resistance exercise did not alter intramuscular adipose tissue but reduced retinolbinding protein-4 concentration in individuals with type 2 diabetes mellitus. J Int Med Res. 2010;38:782-91.

58. Winnick JJ, Gaillard T, Schuster DP. Resistance training differentially affects weight loss and glucose metabolism of white and African American patients with type 2 diabetes mellitus. Ethn Dis. 2008;18:152-6.

59. Madden KM, Lockhart C, Cuff D, Potter TF, Meneilly GS. Short-term aerobic exercise reduces arterial stiffness in older adults with type 2 diabetes, hypertension, and hypercholesterolemia. Diabetes Care. 2009;32:1531-5.

60. Madden KM, Lockhart CK, Potter TF, Cuff DJ, Meneilly GS. Short-term aerobic exercise reduces nitroglycerin-induced orthostatic intolerance in older adults with type 2 diabetes. J Cardiovasc Pharmacol. 2011;57:666-71.

61. Sukala WR, Page R, Rowlands DS, Krebs J, Lys I, Leikis M, Pearce J, Cheema BS. South Pacific islanders resist type 2 diabetes: comparison of aerobic and resistance training. Eur J Appl Physiol. 2012;112:317-25.

62. Kadoglou NP, Iliadis F, Angelopoulou N, Perrea D, Ampatzidis G, Liapis CD, Alevizos M. The anti-inflammatory effects of exercise training in patients with type 2 diabetes mellitus. Eur J Cardiovasc Prev Rehabil. 2007;14:837-43.

63. Alam S, Stolinski M, Pentecost C, Boroujerdi MA, Jones RH, Sonksen PH, Umpleby AM. The effect of a six-month exercise program on very lowdensity lipoprotein apolipoprotein B secretion in type 2 diabetes. J Clin Endocrinol Metab. 2004;89:688-94.

64. Aylin K, Arzu D, Sabri S, Handan TE, Ridvan A. The effect of combined resistance and home-based walking exercise in type 2 diabetes patients. Int J Diabetes Dev Ctries. 2009;29:159-65.

65. de Oliveira VN, Bessa A, Jorge ML. The effect of different training programs on antioxidant status, oxidative stress, and metabolic control in type 2 diabetes. Appl Physiol Nutr Metab. 2012;37:334-44.

66. Jennings AE, Alberga A, Sigal RJ. The effect of exercise training on resting metabolic rate in type 2 diabetes mellitus. Med Sci Sports Exerc. 2009;41: 1558-65.

67. Kwon HR, Han KA, Ku YH, Ahn HJ, Koo BK, Kim HC, Min KW. The effects of resistance training on muscle and body fat mass and muscle strength in type 2 diabetic women. Korean Diabetes J. 2010;34(2):101-10.

68. Cauza E, Hanusch-Enserer U, Strasser B, Ludvik B, Metz-Schimmerl S, Pacini G, Wagner O, Georg P, Prager R, Kostner K, Dunky A, Haber P. The relative benefits of endurance and strength training on the metabolic factors and muscle function of people with type 2 diabetes mellitus. Arch Phys Med Rehabil. 2005;86:1527-33.

69. Caidahl K, Caidahl K, Krook A. Effects of Nordic walking on cardiovascular risk factors in overweight individuals with type 2 diabetes, impaired or normal glucose tolerance. Diabetes Metab Res Rev. 2013;29:25-32.

70. Tessier D, Ménard J, Fülöp T, Ardilouze J, Roy M, Dubuc N, Dubois M, Gauthier P. Effects of aerobic physical exercise in the elderly with type 2 diabetes mellitus. Arch Gerontol Geriatr. 2000;1(31):121-32.

71. Wei M, Gibbons LW, Mitchell TL, Kampert JB, Lee CD, Blair SN. The association between cardiorespiratory fitness and impaired fasting glucose and type 2 diabetes mellitus in men. Ann Intern Med. 1999;130:89-96.

72. Reaven GM. Banting lecture: role of insulin resistance in human disease. Diabetes. 1988;37:1595-607.

73. Betteridge DJ. Diabetic dyslipidaemia. Eur J Clin Investig. 1999;29:12-6.

74. Lehmann R, Vokac A, Niedermann K, Agosti K, Spinas GA. Loss of abdominal fat and improvement of the cardiovascular risk profile by regular moderate exercise training in patients with NIDDM. Diabetologia. 1995;38:1313-9.

75. Garber CE, Blissmer B, Deschenes MR, Franklin BA, Lamonte MJ, Lee IM, et al. American College of Sports Medicine. American College of Sports Medicine position stand. Quantity and quality of exercise for developing and maintaining cardiorespiratory, musculoskeletal, and neuromotor fitness in apparently healthy adults: guidance for prescribing exercise. Med Sci Sports Exerc. 2011;43:1334-59.

76. Gordon BA, Benson AC, Bird SR, Fraser SF. Resistance training improves metabolic health in type 2 diabetes: a systematic review. Diabetes Res Clin Pract. 2009;83:157-75.
77. Kelley GA, Kelley KS. Effects of aerobic exercise on lipids and lipoproteins in adults with type 2 diabetes: a meta-analysis of randomized-controlled trials. Public Health. 2007;121:643-55.

78. Umpierre D, Ribeiro PA, Schaan BD, Ribeiro JP. Volume of supervised exercise training impacts glycaemic control in patients with type 2 diabetes: a systematic review with meta-regression analysis. Diabetologia. 2013;56:242-51.

79. Holton DR, Colberg SR, Nunnold T, Parson HK, Vinik Al. The effect of an aerobic exercise training program on quality of life in type 2 diabetes. The Diabetes Educator. 2003;29:837-46.

80. Bello Al, Owusu-Boakye E, Adegoke BO, Adjei DN. Effects of aerobic exercise on selected physiological parameters and quality of life in patients with type 2 diabetes mellitus. Int J Gen Med. 2011;4:723-7.

81. Fritz T, Caidahl K, Osler M, Ostenson CG, Zierath JR, Wändell P. Effects of Nordic walking on health-related quality of life in overweight individuals with type 2 diabetes mellitus, impaired or normal glucose tolerance. Diabet Med. 2011;(11):1362-72.

\section{Ready to submit your research? Choose BMC and benefit from:}

- fast, convenient online submission

- thorough peer review by experienced researchers in your field

- rapid publication on acceptance

- support for research data, including large and complex data types

- gold Open Access which fosters wider collaboration and increased citations

- maximum visibility for your research: over $100 \mathrm{M}$ website views per year

At BMC, research is always in progress.

Learn more biomedcentral.com/submissions 